\title{
A Model Predictive Control Approach to Trajectory Tracking Problems via Time-Varying Level Sets of Lyapunov Functions
}

\author{
Timm Faulwasser and Rolf Findeisen
}

\begin{abstract}
We discuss a model predictive control approach to trajectory tracking problems of constrained nonlinear continuous time systems, where the reference trajectory is a priori known and asymptotically constant. The proposed NMPC scheme is able to explicitly consider input and state constraints while guaranteeing recursive feasibility. To handle the timevarying nature of the tracking problem we advocate the use of time-varying level sets of Lyapunov functions as terminal regions. We prove a necessary and sufficient condition for positive invariance of these sets and show how these sets can be efficiently computed, if a quadratic Lyapunov function is available. As an example we consider a nonlinear CSTR reactor.
\end{abstract}

Index Terms-nonlinear model predictive control, trajectory tracking, time-varying level sets, invariant sets

\section{INTRODUCTION}

Nonlinear model predictive control (NMPC) is an especially useful control strategy for MIMO systems that are subject to input and state constraints. By now many elaborated results exist for set-point stabilization, both in discrete and continuous time, e.g. [4], [6], [11]. Results on predictive control for path-following problems are also available [5]. So far only limited results for the application of NMPC to problems governed by time-varying dynamics are available, e.g. [6], [8]. One reason why results on NMPC for time-varying systems are rather sparse in the literature are the difficulties of designing local control Lyapunov functions -which are often used to enforce stability via terminal constraints and end penalties- for time-varying dynamics.

In this work we discuss the application of NMPC to constrained trajectory tracking problems, where the reference trajectory is a priori known and asymptotically constant. This leads to inherently time-varying problems. Explicit stability results on NMPC for trajectory tracking are limited. Few works consider tracking problems without constraints: In [9] output tracking is discussed in discrete time, while [12] presents results for continuous time systems based on a terminal equality constraint. The tracking of asymptotically constant references in the presence of constraints has been considered in [10]. There the prediction horizon is required to be longer than the finite convergence time of the reference.

Our contributions are as follows: In Section II we propose a sampled-data continuous time NMPC scheme for trajectory tracking of asymptotically constant references, where input

T. Faulwasser and R. Findeisen are with the Institute for Automation Engineering, Otto-von-Guericke University Magdeburg, Germany. T. Faulwasser is also a member of the International Max Planck Research School for Analysis, Design and Optimization in Chemical and Biochemical Process Engineering Magdeburg. \{timm.faulwasser, rolf.findeisen\}argu.de and state constraints are present. We state sufficient convergence conditions based on time-varying terminal regions and end penalties. In comparison to previous works the proposed NMPC scheme allows choosing the length of the prediction horizon completely independent from the convergence time of the reference, cf. [10]. Hence one can use short horizons which are desirable from the computations point of view. We consider state and input constraints and do not require zero-terminal constraints to guarantee convergence of the tracking error, cf. [9], [12]. Our main results are presented in Section III: We introduce a concept of time-varying level sets of Lyapunov functions and prove a necessary and sufficient condition for positive invariance of these sets. We show how these sets can be obtained via a convex optimization problem, if a quadratic Lyapunov function is available. This allows to guarantee stability of the NMPC scheme and facilitates recursive feasibility of the optimal control problems. In Section IV we consider a trajectory tracking task for a nonlinear chemical reactor as an example. We draw conclusions in Section V.

\section{Notation \& Definitions}

The interior of a compact set $\mathcal{B}$ is denoted as $\operatorname{int}(\mathcal{B})$. The set of $k$-times continuously differentiable functions on $\mathbb{R}$ is written as $\mathcal{C}^{k}$. The norm $\|x\|$ of $x \in \mathbb{R}^{n}$ denotes the 2-norm and $\|A\|$ of $A \in \mathbb{R}^{n \times n}$ the induced 2-norm. The solution at time $t$ of an ODE $\dot{x}=f(x, u)$ starting at $x\left(t_{0}\right)=x_{0}$ and driven by the input $u$ is denoted as $x\left(t, x_{0} \mid u\right)$. If no ambiguity about the initial condition can arise we write $x(t \mid u)$. The set of time-varying matrices in $\mathbb{R}^{n \times m}$ that are piecewise continuous and bounded in their elements for all $t \in[0, \infty)$ is written as $\mathcal{B} C\left(\mathbb{R}^{n \times m}\right)$. If $A(t) \in \mathcal{B} C\left(\mathbb{R}^{n \times n}\right)$ is symmetric and positive semi-definite, then we write $A(t) \in \mathcal{B} C_{0}^{+}\left(\mathbb{R}^{n \times n}\right)$. Accordingly $A(t) \in$ $\mathcal{B} C^{+}\left(\mathbb{R}^{n \times n}\right)$, if $A(t)=A^{T}(t)>0$. The minimal eigenvalue of $Q \in \mathbb{R}^{n \times n}$ is denoted as $\lambda_{\min }(Q)$.

\section{AN NMPC SCHEME FOR TRAJECTORY TRACKING}

Consider a continuous time nonlinear system of the form

$$
\dot{x}(t)=f(x(t), u(t)), \quad x(0)=x_{0},
$$

where $x \in \mathcal{X} \subset \mathbb{R}^{n}$ and $u \in \mathcal{U} \subseteq \mathbb{R}^{m}$ are state and input constraints defined by simply connected compact sets $\mathcal{X}, \mathcal{U}$. We assume that $f$ is continuous and locally Lipschitz in $x$. Subsequently we want to solve the following trajectory tracking problem. 
Problem 1 (Constrained Trajectory Tracking)

Given a system (1) and an a priori known reference trajectory $x_{r}(t) \in \mathcal{C}^{1}$. Design a controller such that:

P1 Reference Convergence: The system state $x(t)$ converges to the reference $\lim _{t \rightarrow \infty}\left\|x(t)-x_{r}(t)\right\|=0$.

P2 Constraint Satisfaction: The state and input constraints are satisfied for all $t \geq 0: x(t) \in \mathcal{X}, u(t) \in \mathcal{U}$.

The standard approach to solve trajectory tracking problems is based on the definition of the error variable

$$
e(t):=x(t)-x_{r}(t) .
$$

Under the assumptions that the reference is a priori known and $x_{r}(t) \in \mathcal{C}^{1}$ the tracking problem can be reformulated as a set-point stabilization problem of the error dynamics

$$
\dot{e}=\tilde{f}(t, e, u):=f\left(e+x_{r}(t), u\right)-\dot{x}_{r}(t),
$$

which are inherently time-varying for varying $x_{r}(t)$.

In order to solve this problem we propose an NMPC scheme based on the error dynamics (3). To distinguish between the predicted controller variables and the real system variables we denote the predicted inputs and states with $\bar{e}, \bar{u}$ etc. To find suitable inputs, as usual in NMPC, at each sampling instant $t_{k}=k \delta, \quad \delta>0, k \in \mathbb{N}$ the following optimal control problem (OCP) is solved repeatedly

$$
\underset{\bar{u}(\cdot)}{\operatorname{minimize}} J\left(e\left(t_{k}\right), \bar{e}(\cdot), \bar{u}(\cdot)\right) .
$$

The cost functional to be minimized is given as

$$
J(\cdot)=\int_{t_{k}}^{t_{k}+T_{p}} F(t, \bar{e}, \bar{u}) d \tau+\left.E(t, \bar{e})\right|_{t_{k}+T_{p}},
$$

where $F(\cdot): \mathbb{R}_{0}^{+} \times \mathcal{X} \times \mathcal{U} \rightarrow \mathbb{R}_{0}^{+}$is denoted as stage cost and $F(t, 0,0)=0$. We assume that $F$ is lower bounded by a class $\mathcal{K}$ function $\psi(\|e\|)$. As standard in NMPC the term $E(\cdot): \mathbb{R}_{0}^{+} \times \mathbb{R}^{n} \rightarrow \mathbb{R}_{0}^{+}$is referred to as end penalty, see [11]. The time $T_{p}$ denotes the prediction horizon. This optimal control problem is subject to the predicted system behavior and further constraints

$$
\begin{aligned}
\dot{\bar{e}}=\tilde{f}(t, \bar{e}, \bar{u}), & \bar{e}\left(t_{k}\right)=x\left(t_{k}\right)-x_{r}\left(t_{k}\right), \\
\forall t \in\left[t_{k}, t_{k}+T_{p}\right]: \quad & \bar{e}(t)+x_{r}(t) \in \mathcal{X}, \bar{u}(t) \in \mathcal{U}, \\
& \bar{e}\left(t_{k}+T_{p}\right) \in \mathcal{E}_{t_{k}+T_{p}} .
\end{aligned}
$$

Finally the optimal solution $\bar{u}^{\star}\left(t \mid \bar{e}\left(t_{k}\right)\right)$ is applied to the system such that for all $t \in\left(t_{k}, t_{k+1}\right]: u(t)=\bar{u}^{\star}(\cdot)$. For simplicity we assume that the problem data guarantees the existence of an optimal solution which is attained, cf. [6].

Here we advocate the use of time-varying terminal regions, which we denote as $\mathcal{E}_{t_{k}+T_{p}}$. Therefore (4e) requires that at the end of each prediction the predicted tracking error $\bar{e}\left(t_{k}+\right.$ $T_{p}$ ) has to be inside a time-varying terminal region. Clearly for all $e \in \mathcal{E}_{t_{k}+T_{p}}: e+x_{r}\left(t_{k}+T_{p}\right) \in \mathcal{X}$ needs to hold. In Section III we show how suitable sets $\mathcal{E}_{t}$ can be derived via the concept of time-varying level sets of a Lyapunov function. Note that the length of the prediction horizon $T_{p}$ has to be chosen such that the OCP (4) is feasible.
As mentioned before the trajectory tracking problem is inherently time-varying, which needs to be taken into account, if one wants to derive stability conditions. Hence the next result is mainly an adaption of NMPC stability results presented in [6].

Theorem 1 (Convergence of NMPC for Trajectory Tracking) Consider the constrained trajectory tracking problem (P1P2) for system (1).

Suppose that an end penalty $E(t, e(t)) \in \mathcal{C}^{1}$ and a timevarying terminal region $\mathcal{E}_{t}$ exist such that for all $t \geq 0 a$ control $u(t)=k(t, e) \in \mathcal{U}$ guarantees the following:

- For all $\tilde{e} \in \mathcal{E}_{\tilde{t}}$ and all $t \geq \tilde{t}$

$$
\begin{array}{rll}
e(t, \tilde{e} \mid k(t, e)) & \in \mathcal{E}_{t} \\
x_{r}(t)+e(t, \tilde{e} \mid k(t, e)) & \in \mathcal{X}
\end{array}
$$

hold.

- For all solutions $e(t, \tilde{e} \mid k(t, e)) \in \mathcal{E}_{t}$ it holds

$$
\dot{E}(t, e)+\frac{\partial E(t, e)}{\partial e} \dot{e}+F(t, e, k(t, e)) \leq 0 .
$$

- The optimal control problem (4) has a feasible solution for $e(0)=x_{0}-x_{r}(0)$.

Then the closed loop defined by (1) and (4) guarantees convergence of the tracking error

$$
\lim _{t \rightarrow \infty}\|e(t)\|=\lim _{t \rightarrow \infty}\left\|x(t)-x_{r}(t)\right\|=0
$$

and constraint satisfaction $e(t)+x_{r}(t) \in \mathcal{X}, u(t) \in \mathcal{U}$.

The proof of the theorem employs the ideas on NMPC for time-varying systems as presented in [6]. We sketch briefly the main issues here. Firstly, note that (5a) is simply a positive invariance condition, where the invariant set $\mathcal{E}_{t}$ is allowed to change with time. Secondly, recursive feasibility can easily be shown by concatenating an optimal input with the terminal feedback law $k(t, e)$. Thirdly, condition (5b) requires a cost decrease for all $e(t) \in \mathcal{E}_{t}$, which is necessary in order to use the value function of the OCP (4) as a Lyapunov function. The main difference to standard approaches is that the end penalty $E(t, e)$ as well as the terminal region $\mathcal{E}_{t}$ may depend explicitly on time, cf. [6], [11]. This theorem is the basis for the further developments.

\section{Suitable Terminal Regions and End Penalties}

Subsequently we discuss how a suitable time-varying terminal region $\mathcal{E}_{t}$ and an end penalty $E(\cdot)$ can be determined such that the suppositions of Theorem 1 are fulfilled. Our agenda is as follows: Firstly, we consider the linearization of the error dynamics (3) along the reference $x_{r}(t)$. We derive a suitable feedback as well as a Lyapunov function for the contolled linear time-varying (LTV) error system. This feedback law serves as terminal control law $k(t, e)$ in (5). Secondly, we introduce the concept of time-varying level sets of Lyapunov functions. We formulate an optimal control problem to compute constraint consistent time-varying level sets for the LTV error system. We show how this proposed problem can an be approximated as a convex optimization problem. Based on these time-varying sets we derive a 
suitable terminal region $\mathcal{E}_{t}$ for the nonlinear error dynamics. Finally, we clarify how the conditions of Theorem 1 can be verified.

To follow this agenda we make standing assumptions.

A1 The a priori known reference trajectory is asymptotically constant and defined for all $t \geq 0$, s.t. $x_{r}(t) \in$ $\operatorname{int}(\mathcal{X}) \subset \mathbb{R}^{n}$ and $x_{r}(t) \in \mathcal{C}^{1}$. Furthermore $\lim _{t \rightarrow \infty} x_{r}(t)=$ $x_{r}(T)$ with $T<\infty$.

A2 An admissible reference input $u_{r}(t) \in \operatorname{int}(\mathcal{U}) \subset \mathbb{R}^{m}$ defined for all $t \geq 0$ with $\dot{x}_{r}=f\left(x_{r}, u_{r}\right)$ is known.

A3 The reference trajectory $x_{r}(t)$ ends in a stabilizable setpoint of (1). The matrices $A(T), B(T)$

$$
A(T)=\left.\frac{\partial f}{\partial x}\right|_{x_{r}(T), u_{r}(T)}, B(T)=\left.\frac{\partial f}{\partial u}\right|_{x_{r}(T), u_{r}(T)}
$$

are stabilizable and $\left.f(x, u)\right|_{x_{r}(T), u_{r}(T)}=0$.

A4 The constraints $\mathcal{X}, U$ of system (1) are box constraints $x_{\min } \leq x \leq x_{\max }$ and $u_{\min } \leq u \leq u_{\max }$.

A5 The stage cost $F$ in (4b) is

$$
F(t, e, u):=e^{T} Q_{F} e+\left(u-u_{r}(t)\right)^{T} R_{F}\left(u-u_{r}(t)\right)
$$

with $Q_{F}, R_{F}>0$.

\section{A. Stabilization of the LTV Error System}

Consider the error dynamics (3) and assumptions A1A3. The Jacobian linearization of the error system along the reference trajectory $x_{r}(t)$ and reference input $u_{r}(t)$ expressed in the coordinates $e=x-x_{r}$ and $w=u-u_{r}$ is

$$
\dot{e}=A(t) e+B(t) w,
$$

where the time-varying matrices $A(t) \in \mathcal{B} C\left(\mathbb{R}^{n \times n}\right)$ and $B(t) \in \mathcal{B} C\left(\mathbb{R}^{n \times m}\right)$. The next lemma states sufficient conditions for exponential stabilizability of the LTV system (7).

Lemma 1 (Exponential stabilizability of LTV error system) If assumptions A1-A3 hold, then the LTV error system (7) is exponentially stabilizable.

Proof. We adapt results on the exponential stabilizability of LTV systems, cf. [13] and give a constructive proof. Recall that $A(t) \in \mathcal{B} C\left(\mathbb{R}^{n \times n}\right)$ and $B(t) \in \mathcal{B} C\left(\mathbb{R}^{n \times m}\right)$. W.l.o.g. assume that two matrices $Q(t) \in \mathcal{B} C^{+}\left(\mathbb{R}^{n \times n}\right)$ and $R(t) \in$ $\mathcal{B} C^{+}\left(\mathbb{R}^{m \times m}\right)$ are choosen such that for all $t \in[0, T]$

$$
Q(t)-\left(A(t)+A^{T}(t)+B(t) R^{-1}(t) B^{T}(t)\right) \geq \tilde{q} I
$$

holds for some $\tilde{q}>0$ and for $t \geq T$ the matrices $Q(t)$ and $R(t)$ are constant. We subsequently verify that the timevarying feedback given by

$$
\omega=K(t) e=-\frac{1}{2} R^{-1}(t) B^{T}(t)(P(t)-I) e
$$

stabilizes the LTV error system (7) exponentially. Here $P(t) \in \mathcal{B} C_{0}^{+}\left(\mathbb{R}^{n \times n}\right)$ is the solution to the Riccati differential equation (RDE)

$$
\begin{aligned}
\dot{P}(t) & =P(t) B(t) R^{-1}(t) B^{T}(t) P(t)-Q(t) \\
& -P(t) A(t)-A^{T}(t) P(t), \quad P(T)=P_{T} \geq 0 .
\end{aligned}
$$

From the fact that for $t \geq T$ the LTV error system is stabilizable the existence of a positive semi-definite solution to (10) such that for all $t \geq 0: P(t) \in \mathcal{B} C_{0}^{+}\left(\mathbb{R}^{n \times n}\right)$ can be inferred, cf. [1], [13].

Denote as $\tilde{A}(t):=A(t)+B(t) K(t)$ the closed loop system matrix of (7) under (9). Consider the candidate Lyapunov function

$$
V(t, e)=e^{T}(P(t)+I) e
$$

where $P(t)+I \in \mathcal{B} C^{+}\left(\mathbb{R}^{n \times n}\right)$. Its time derivative along the solution trajectories of $(7)$ is

$$
\dot{V}=e^{T}\left(A(t)+A^{T}(t)+B(t) R^{-1}(t) B^{T}(t)-Q(t)\right) e
$$

It follows from (8) that $\dot{V}(t, e) \leq-\tilde{q}\|e\|^{2}$. Hence $V(t, e):=$ $e^{T}(P(t)+I) e$ is a quadratic Lyapunov function for (7) under the feedback (9). Hence the LTV error system (7) is exponentially stabilizable.

Remark 1 (Choosing Weight Matrices for RDE)

Equation (8) seems to be quite hard to verify. However, for chosen constant $R(t)=R>0$ one can set

$$
Q(t):=\tilde{Q}+A(t)+A^{T}(t)+B(t) R^{-1} B^{T}(t)
$$

and determine a suitable constant matrix $\tilde{Q}>0$ via a timediscretized LMI approximation of (8). In that case the decay (12) of $V(t, e)$ is $\dot{V}=-e^{T} \tilde{Q} e$ and for all $t \geq T$ the $R D E$ (10) is time-invariant. Hence the boundary condition $P_{T} \geq 0$ should be obtained as stationary solution of (10) at $t=T$. This also ensures that $V(\cdot)$ is defined for all $t \geq 0$.

\section{B. Invariant Time-Varying Sets}

In standard quasi-infinite horizon NMPC approaches ([4], [6]) one often computes a suitable level set $\mathcal{E}=\{e \in$ $\left.\mathbb{R}^{n} \mid V(t, e) \leq c^{2}\right\}$ such that for all $e \in \mathcal{E}$ conditions similar to (5) hold. This is restrictive, since the level set is defined by a constant. Subsequently it is our main idea to relax this conservatism by time-varying levels defined by an inequality $V(t, e) \leq \pi^{2}(t)$. In order to do so we introduce the concept of positive invariant time-varying level sets of a Lyapunov function.

Consider the Lyapunov function (11) for the LTV system and a function $\pi \in \mathcal{C}^{1}: \mathbb{R}_{0}^{+} \rightarrow \mathbb{R}^{+}$. In order to handle the time-varying right hand side in the inequality $V(t, e) \leq$ $\pi^{2}(t)$ we consider the closed set

$$
\Lambda:=\left\{(t, e) \mid V(t, e) \leq \pi^{2}(t)\right\} \subset \mathbb{R}_{0}^{+} \times \mathbb{R}^{n}
$$

in the extended space $\mathbb{R}_{0}^{+} \times \mathbb{R}^{n}$. If the time coordinate of $\Lambda$ is fixed we write

$$
\Lambda_{t}:=\Lambda \cap\left\{\{t\} \times \mathbb{R}^{n}\right\} .
$$

Additionally we define a projection $\Pi: \mathbb{R}_{0}^{+} \times \mathbb{R}^{n} \rightarrow \mathbb{R}^{n}$

$$
\Pi:(t, e) \mapsto e \in \mathbb{R}^{n}
$$

which continuously maps any extended state $(t, e)$ onto $e$. The images $\Pi\left(\Lambda_{t}\right)$ are the time-varying level sets. 
Definition 1 (Time-Varying Level Sets)

For all $t \geq 0$ we call the family of subsets of $\mathbb{R}^{n}$ given by

$$
\mathcal{L}_{t, V, \pi}:=\left\{e \in \mathbb{R}^{n} \mid(t, e) \in \Lambda\right\}=\Pi\left(\Lambda_{t}\right)
$$

a time-varying level set of the Lyapunov function $V(t, e)$ from (11). Accordingly we define the boundary of $\mathcal{L}_{t, V, \pi}$ pointwise in time as

$$
\partial \mathcal{L}_{t, V, \pi}:=\partial \Pi\left(\Lambda_{t}\right) .
$$

For the LTV error system of the trajectory tracking task, the time-varying level set are ellipsoids $\left\{x \in \mathbb{R}^{n} \mid(x-\right.$ $\left.\left.x_{r}(t)\right)^{T}(P(t)+I)\left(x-x_{r}(t)\right) \leq \pi^{2}(t)\right\}$ which are centered along the reference trajectory $x_{r}(t)$.

For time-invariant level sets $\left\{V(t, e) \leq c^{2}\right\}$ it is straightforward to conclude positive invariance. In the case of timevarying level sets as defined above this question is more complicated to answer.

Theorem 2 (Positive Invariance of Time-Varying Level Sets) Consider system (7) under the feedback (9) and the sets $\mathcal{L}_{t, V, \pi}=\Pi\left(\Lambda_{t}\right)$ from (17) where for all $t \geq 0: \pi(t)>0$.

All solutions $e\left(t, e_{0} \mid K(t) e\right)$ with $e_{0} \in \mathcal{L}_{t=0, V, \pi}$ stay inside $\mathcal{L}_{t, V, \pi}$ for all $t \geq 0$ if and only if

$$
\forall t \geq 0, \forall e \in \partial \mathcal{L}_{t, V, \pi}: \quad \dot{V}(t, e) \leq 2 \pi(t) \dot{\pi}(t) .
$$

Proof. Our proof is based on Nagumos Theorem on positive invariance of sets for time-invariant systems, cf. [2].

We begin with proving the sufficiency of (18). The main idea is to verify that (18) ensures the positive invariance of the set $\Lambda$ under the dynamics of the augmented system

$$
\left(\begin{array}{c}
\dot{t} \\
\dot{e}
\end{array}\right)=\left(\begin{array}{c}
1 \\
\tilde{A}(t) e
\end{array}\right), \quad \tilde{A}(t)=A(t)+B(t) K(t) .
$$

Under the change of variables $z:=(t, e)^{T}$ the set $\Lambda$ is described as $\left\{z \in \mathbb{R}_{0}^{+} \times \mathbb{R}^{n} \mid v(z) \leq 0\right\}$ where $v(z)=$ $V(z)-\pi^{2}(z)$. Note that $\Lambda$ is a closed set. The tangent cone of $\Lambda$ at any $z \in \partial \Lambda$ with is

$$
\mathcal{T}_{\Lambda}(z)=\left\{\zeta \in \mathbb{R}^{1+n} \mid \nabla v(z)^{T} \zeta \leq 0\right\} .
$$

According to Nagumos Theorem positive invariance of $\Lambda$ is verified if the velocity vector $\dot{z}$ is contained in $\mathcal{T}_{\Lambda}(z)$ for all $z \in \partial \Lambda$. We verify this in $(t, e)$ coordinates and obtain

$$
\left(\begin{array}{c}
\frac{d V(t, e)}{d t}-2 \pi \dot{\pi} \\
\frac{\partial V(t, e)}{\partial e}
\end{array}\right)^{T}\left(\begin{array}{c}
1 \\
\tilde{A}(t) e
\end{array}\right)=\dot{V}(t, e)-2 \pi \dot{\pi} .
$$

Hence for all $z \in \partial \Lambda$ supposition (18) ensures that $\dot{z}=$ $(1, \tilde{A}(t) e)^{T} \in \mathcal{T}_{\Lambda}(z)$. It follows that $\Lambda$ is an invariant set of the augmented system (19). Due to the construction of $\mathcal{L}_{t, V, \pi}$ via $\Pi$ from (16) this directly implies that if $e\left(t_{0}\right) \in \mathcal{L}_{t_{0}, V, \pi}$ with $t_{0} \geq 0$ then for all $t \geq t_{0}: e\left(t, e\left(t_{0}\right) \mid k(t, e)\right) \in \mathcal{L}_{t, V, \pi}$.

The necessity of (18) is verified as follows. Assume that for some $z_{0}=\left(t_{0}, e_{0}\right) \in \partial \Lambda$ with $t_{0} \geq 0$ we have $\dot{z}_{0} \notin$ $\mathcal{T}_{\Lambda}\left(z_{0}\right)$. Using the definition of the tangent cone (20) we have that $\nabla v\left(z_{0}\right) \dot{z}_{0}>0$. Hence $\left.\frac{d v(z)}{d t}\right|_{z_{0}}=\nabla v\left(z_{0}\right) \dot{z}_{0}>0$, which implies that for some $\tau \in\left[t_{0}, t_{0}+h\right]$ we have that $v(z(\tau))>0$. Consequently $\dot{z}_{0} \notin \mathcal{T}_{\Lambda}\left(z_{0}\right)$ implies $z(\tau) \notin \Lambda$ and $e\left(\tau, e\left(t_{0}\right) \mid k(\tau, e)\right) \notin \mathcal{L}_{\tau, V, \pi}$.

\section{Computation of Time-Varying Level Sets}

Naturally, the question arises how to determine $\pi(t)$ such that $\mathcal{L}_{t, V, \pi}$ based on $V$ from (11) is as large as possible, consistent with the constraints, and positive invariant in the sense of Theorem 2 .

To achieve this one can state the problem of finding the maximum volume constraint consistent positive invariant time-varying level set as follows.

$$
\underset{w_{\pi}, \pi_{0}}{\operatorname{maximize}} \int_{0}^{T} \operatorname{vol}\left(\mathcal{L}_{\tau, V, \pi}\right) d \tau
$$

subject to the dynamics

$$
\dot{\pi}(t)=w_{\pi}(t), \quad \pi(0)=\pi_{0}, \dot{\pi}(T)=0
$$

and the following constraints

$$
\begin{aligned}
& \forall t \in[0, T]: \quad \pi(t)>0, \\
& \forall t \in[0, T]: \quad \mathcal{L}_{t, V, \pi}=\Pi\left(\Lambda_{t}\right), \\
& \forall e \in \partial \mathcal{L}_{t, V, \pi}: \quad \dot{V}(t, e) \leq 2 \pi(t) w_{\pi}(t), \\
& \forall e \in \mathcal{L}_{t, V, \pi}: \quad K(t) e+u_{r}(t) \in \mathcal{U} \subset \mathbb{R}^{m}, \\
& \forall e \in \mathcal{L}_{t, V, \pi}: \quad e+x_{r}(t) \in \mathcal{X} \subseteq \mathbb{R}^{n} \text {. }
\end{aligned}
$$

The main idea behind this optimization problem is to maximize the volume of the corresponding projections $\mathcal{L}_{t, V, \pi}=$ $\Pi\left(\Lambda_{t}\right)$ over the time span $[0, T]$. The time evolution of $\pi(t)$ is described by the scalar ODE (21b), where $w_{\pi}$ is the input and consequently a decision variables of the OCP (21). At the end of the reference trajectory the time-varying level set should not change anymore, hence for $t=T$ we require $\dot{\pi}(T)=0$. The initial condition $\pi(0)=\pi_{0}$ is also a decision variable. Due to the construction of the time-varying level sets $\pi(t)$ has to be always strictly positive (21c). The positive invariance property from (18) is expressed in (21e). Naturally, we want to achieve that the constraints on states and input are respected, hence (21f-g).

The optimal control problem (21) is not straightforward to solve. Relying on concepts for computation of maximum volume ellipsoids we reformulate (21) as a convex problem, cf. [3]. Consider the change of coordinates $\xi=$ $\frac{1}{\pi(t)} S(t) e, \quad S(t)=\sqrt{(P(t)+I)}$. Rewriting $\mathcal{L}_{t, V, \pi}$ in new $\xi$ coordinates yields $\mathcal{L}_{t, V, \pi}=\left\{\pi(t) S^{-1}(t) \xi \mid\|\xi\|^{2} \leq 1\right\}$. Relying on this reformulation the objective (21a) can be stated as maximize $\int_{0}^{T} \log \operatorname{det}\left(S^{-1}(\tau) \pi(\tau)\right) d \tau$. The evolution of $P(t)$ and hence also the evolution of $S(t)$ are not effected by the choice of $\pi(t)$ hence the objective (21a) is equivalent to maximizing $\int_{0}^{T} \pi(t)$. If the weight matrix $Q(t)$ is choosen according to Remark 1 we have $\dot{V}(t, e)=$ $-e^{T} \tilde{Q} e$. Rewriting this in $\xi$ coordinates yields $\dot{V}(t, e)=$ $-\pi^{2}(t) \cdot \xi^{T} S^{-T}(t) \tilde{Q} S^{-1}(t) \xi \leq-\underline{q}(t) \pi^{2}(t)$, where $\underline{q}(t)=$ $\lambda_{\min }\left(S^{-T}(t) \tilde{Q} S^{-1}(t)\right)>0$. Since $\pi(t)>0, \dot{\pi}=\bar{w}_{\pi}$ we require $-q(t) \pi(t) \leq 2 w_{\pi}(t)$ instead of $(21 \mathrm{e})$.

Using similar ideas for (21f-g) the reformulation of (21) can now be stated as follows.

$$
\underset{w_{\pi}, \pi_{0}}{\operatorname{maximize}} \int_{0}^{T} \pi(\tau) d \tau
$$


subject to the linear dynamics

$$
\dot{\pi}(t)=w_{\pi}(t), \quad \pi(0)=\pi_{0}, \dot{\pi}(T)=0,
$$

and additional constraints

$$
\begin{aligned}
2 w_{\pi}(t) & \geq-\pi(t) \cdot \underline{q}(t), \\
\left\|x_{i}-x_{r}(t)\right\| & \geq \pi(t) \cdot\left\|S^{-1}(t)\right\|, \\
\left\|u_{i}-u_{r}(t)\right\| & \geq \pi(t) \cdot\left\|K(t) S^{-1}(t)\right\|,
\end{aligned}
$$

where $\underline{q}(t)=\lambda_{\min }\left(S^{-T}(t) \tilde{Q} S^{-1}(t)\right)$ and $i \in\{\min , \max \}$. Note that $\pi(t)$ and $w_{\pi}(t)$ appear in affine expressions. Hence a time discretization of (22) leads to a linear programm.

\section{Terminal Regions and End Penalties}

We need to ensure that the time-varying set $\mathcal{L}_{t, V, \pi}$ is positive invariant under the nonlinear error dynamics (3) subject the two degrees of freedom control $u=K(t) e+$ $u_{r}(t)$. In general this is not the case and difficult to enforce. However, we know that the closed loop LTV error system (7) is (exponentially) stable. Hence it can be shown that for some $0<\tilde{\pi}(t) \leq \pi(t)$ the set $\tilde{\mathcal{L}}_{t, V, \tilde{\pi}}$ will be positive invariant for the nonlinear error system (3) controlled via $u=K(t) e+u_{r}(t)$. To obtain $\tilde{\pi}(t)$ we rewrite the nonlinear error dynamics (3)

$$
\dot{e}=\tilde{A}(t) e+\Phi(t, e)
$$

where $\tilde{A}(t)=A(t)+B(t) K(t)$ is the closed loop system matrix of the LTV error system and $\Phi(t, e)=$ $\tilde{f}\left(t, e, K(t) e+u_{r}(t)\right)-\tilde{A}(t) e$.

Actually, we need to guarantee that for all $t \geq T$ the Lyapunov function of the LTV error system is also a local Lyapunov function for the nonlinear error dynamics valid on $\tilde{\mathcal{L}}_{t, V, \tilde{\pi}}$. It is important to note that for the preceding time points $t \in[0, T)$ positive invariance of $\tilde{\mathcal{L}}_{t, V, \tilde{\pi}}$ is sufficient in order to use $\mathcal{L}_{t, V, \tilde{\pi}}$ as terminal region in the NMPC algorithm for trajectory tracking (4). We apply a similar ratio as for usual time-invariant level sets, cf. [4]. We assume that $\pi(t)$ for the LTV error system is computed via a time discretization of (22). We shrink $\pi(t)$ point-wise in time down to $\tilde{\pi}(t) \leq \pi(t)$ by a sequence of global optimization problems. For $t=T$ consider $\tilde{\mathcal{L}}_{T, V, \tilde{\pi}}$ and the function $\phi_{T}$

$$
\phi_{T}(e)=2 e^{T} \tilde{P}(T) \Phi(T, e)-e^{T} \tilde{q} e+\epsilon \cdot e^{T} \tilde{P}(T) e
$$

where $\epsilon=\lambda_{\min }\left(\left.S^{-1}\left(Q_{F}+K^{T} R_{F} K\right) S^{-1}\right|_{t=T}\right)$ and $\tilde{P}(T)=P(T)+I$. Note $\phi_{T}(e)$ is an upper bound for $\frac{d V(t, e)}{d t}$ along the trajectories of the nonlinear error dynamics (3) under the linear feedback (9). Now one iteratively solves $\phi_{T}^{*}=\max \phi_{T}(e)$ for all $e \in \tilde{\mathcal{L}}_{T, V, \tilde{\pi}}$. Start with $\tilde{\pi}(T)=\pi(T)$ and compute $\phi_{T}^{*}$. If $\phi_{T}^{*} \geq 0$ halve $\tilde{\pi}(T)$ and repeat until $\phi_{T}^{*}<0$. This is a nonlinear global optimization problem. For the remaining time points $0 \leq t<T$ we solve the easier problem $\max \phi_{t}(e)$ for all $e \in \partial \tilde{\mathcal{L}}_{t, V, \tilde{\pi}}$ where $\phi_{t}$ is

$$
\phi_{t}(e)=2 e^{T} \tilde{P}(t) \Phi(t, e)-e^{T} \tilde{q} e-2 \dot{\tilde{\pi}}(t) \tilde{\pi}(t) .
$$

Obviously we cannot solve this problem for all $t \in[0, T)$. If one applies time discretization on $\dot{\tilde{\pi}}(t)=w_{\tilde{\pi}}$ a sequence of nonlinear optimization problems is obtained, which can be solved recursively backwards in time. However, one needs to ensure a sufficiently fine time discretization.

It remains to show that the time-varying level sets $\tilde{\mathcal{L}}_{t, V, \tilde{\pi}}$ and a suitably defined end penalty fulfill the suppositions of Theorem 1. The following lemma holds.

\section{Lemma 2 (Stabilizing Time-Varying Level Sets)}

Consider the constrained trajectory tracking problem P1-P2 for system (1). Suppose that for all $t \in[0, T]: \tilde{\pi}(t) \in(0, \infty)$ and $\mathcal{E}_{t_{k}+T_{p}}=\tilde{\mathcal{L}}_{t_{k}+T_{p}}, V, \tilde{\pi}$. The set $\tilde{\mathcal{L}}_{t, V, \tilde{\pi}}$ and the end penalty

$$
E(t)= \begin{cases}\int_{t}^{T} \alpha(\tau) d \tau+\beta \frac{\alpha(T)}{2 \gamma} & t \in[0, T), \\ \beta \frac{\alpha(T)}{2 \gamma} e^{-2 \gamma(t-T)} & t \geq T,\end{cases}
$$

where $\alpha(t), \beta, \gamma$ are

$$
\begin{aligned}
\alpha(t) & =\tilde{\pi}^{2}(t)\left\|S^{-1}(t)\right\|^{2}\left\|Q_{F}+K(t)^{T} R_{F} K(t)\right\|, \\
\beta & =\bar{p}+1, \quad \bar{p}=\sup \|P(t)\|, \\
\gamma & =\frac{1}{2(\bar{p}+1)} \lambda_{\min }\left(\left.S^{-1}\left(Q_{F}+K^{T} R_{F} K\right) S^{-1}\right|_{t=T}\right)
\end{aligned}
$$

fulfill the suppositions of Theorem 1.

Due to space limitations we sketch only the main ideas of the proof here. Clearly the time-varying sets $\tilde{\mathcal{L}}_{t, V, \tilde{\pi}}$ are positive invariant under the terminal control law $u=K(t) e+$ $u_{r}(t)$. Note that state and input constraints are fulfilled for any nominal solution $e\left(t, e_{0} \mid u\right)$ with $e_{0} \in \tilde{\mathcal{L}}_{t, V, \tilde{\pi}}$.

The end penalty (25) is obtained as an approximation of the worst case cost inside $\tilde{\mathcal{L}}_{t, V, \tilde{\pi}}$ which consists of two parts. The first part is the cost associated to a solution which travels through the boundary of the terminal region $\partial \tilde{\mathcal{L}}_{t, V}, \tilde{\pi}$ for all $t \in[0, T]$. The second part uses the fact that inside $\tilde{\mathcal{L}}_{T, V, \tilde{\pi}}$ the terminal control law guarantees exponential cost decrease. Simple calculations verify that $E(t) \in \mathcal{C}^{1}$ and that $E(t)$ fulfills (5b).

\section{TRajectory Tracking of A Chemical Reactor}

As an example we consider a set-point change of a continuously stirred tank reactor (CSTR) along an a priori given reference trajectory. In the reactor an exothermic, irreversible reaction $A \rightarrow B$ takes place. The dynamics of the CSTR are as follows

$$
\begin{aligned}
\dot{c}_{A} & =\frac{q}{V}\left(c_{A f}-c_{A}\right)-k\left(c_{A}, T\right), \\
\dot{T} & =\frac{q}{V}\left(T_{f}-T\right)+\frac{-\triangle H}{\rho C_{p}} k\left(c_{A}, T\right)+\frac{U A}{V \rho C_{p}}\left(T_{c}-T\right),
\end{aligned}
$$

where $k\left(c_{A}, T\right)=k_{0} e^{\frac{-E}{R T}} c_{A}$. Details on the model and its parameters can be found in [7]. The states $c_{A}$ in [mol/l] and $T$ in $[\mathrm{K}]$ describe the concentration of reactant $A$ and the reactor temperature. The coolant stream temperature $T_{c}$ in $[\mathrm{K}]$ is the input variable. The objective is to design an NMPC controller which stabilizes the CSTR around a previously computed reference trajectory which drives the system in 18 minutes from the set-point $c_{A 1}=0.6, T_{1}=344$ to $c_{A 2}=$ $0.45, T_{2}=352$ via the coolant stream temperature $T_{c}$. The system is subject to the input constraint $270 \leq T_{c} \leq 350$ and the state constraints $0.1 \leq c_{A} \leq 0.63,300 \leq T \leq 400$. 


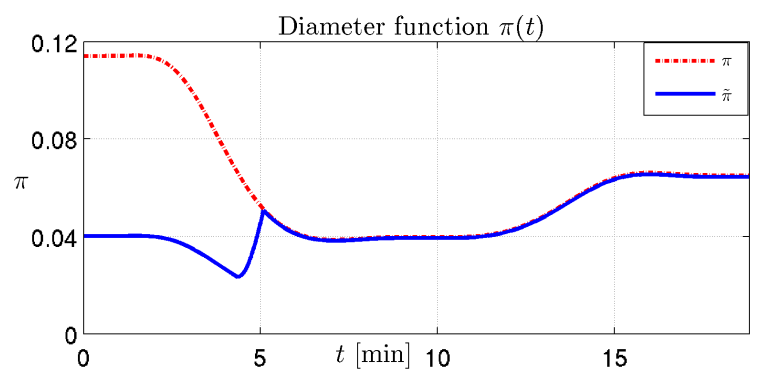

Time-varying level set in $c_{A}-T$ plane

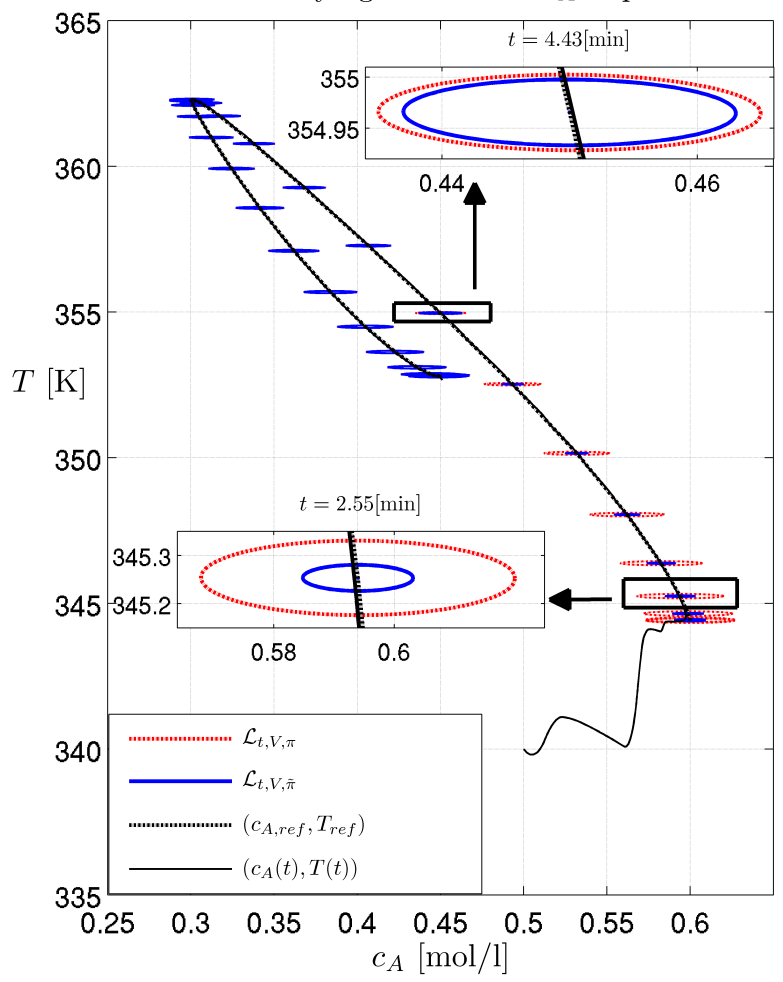

Fig. 1. Visualization of time-varying level sets $\tilde{\mathcal{L}}_{t, V, \tilde{\pi}}$.

The weight matrices for the cost function $F(\cdot)$ are $R_{F}=$ $5 \cdot 10^{-3}$ and $Q_{F}=[60.7,-6.5 ;-6.5,2.5]$. The boundary condition $P(T)$ and the time-varying $Q(t)$ for the RDE (10) are chosen according to Remark 1 with $\tilde{Q}=Q_{F}$ and $R=$ $0.2 \cdot R_{F}$. The prediction horizon is set to $T_{p}=0.5[\mathrm{~min}]$ and much shorter than the length of the reference trajectory which is $18[\mathrm{~min}]$. The control horizon is $\delta=0.3[\mathrm{~min}]$. The diameter functions $\pi(t), \tilde{\pi}(t)$ are obtained via (22) and (24). Both are computed on a time grid with $\delta t=10^{-3}[\mathrm{~min}]$.

The results are illustrated in Figure 1. In the upper part the red dash-dot curve shows $\pi(t)$ which defines the positively invariant time-varying level set $\mathcal{L}_{t, V, \pi}$ from (14) - (17). The blue curve depicts $\tilde{\pi}(t)$ which is obtained via successive shrinking of $\pi(t)$ point-wise in time until (24) is satisfied. The peaks (at $t=4.3$ and $t=5$ ) are caused by changes in the set of active constraints. It should be noted that the time-varying level sets are locally changing in size. This can be observed in Figure 1 upper part, as $\tilde{\pi}(t)$ is increasing along several parts of the reference trajectory. In Figure 1, lower part, the black curve depicts the reference trajectory in the $c_{A}-T$ plane. The red dash-dot ellipsoids are a selection from the time-varying level set of the LTV error system. The blue ellipsoids are samples from the terminal region $\tilde{\tilde{\mathcal{L}}}_{t, V, \tilde{\pi}}$ of the OCP (4). Note we plot only a few of the numerous ellipsoids computed on the time grid with $\delta t=10^{-3}[\mathrm{~min}]$. The thin black line shows one sample trajectory under the proposed NMPC scheme which starts outside of the level set at $t=0$ at $c_{A}=0.5[\mathrm{~mol} / 1], T=340[\mathrm{~K}]$.

\section{Conclusions}

We propose an NMPC scheme for constrained trajectory tracking problems and present convergence conditions where a time-varying terminal region is used. The nice feature about this time-varying terminal regions is that they are not strictly shrinking with time but rather can be locally expanding. In order to achieve this, we introduce the concept of a timevarying level set of a Lyapunov function which is efficiently computable via a convex optimization problem, if a quadratic Lyapunov function is available.

\section{ACKNOWLEDGMENTS}

The authors gratefully acknowledge the helpful discussions with Philipp Rumschinski, Benjamin Kern, Markus Kögel and Friedrich von Haeseler.

\section{REFERENCES}

[1] H. Abou-Kandil, G. Freiling, G. Jank, and V. Ionescu. Matrix Riccati equations in control and systems theory. Systems \& Control: Foundations \& Applications. Birkhäuser, Basel, 2003.

[2] F. Blanchini and S. Miani. Set-theoretic methods in control. Systems \& Control: Foundations \& Applications. Birkhäuser, Basel, 2008.

[3] S.P. Boyd and L. Vandenberghe. Convex Optimization. University Press, Cambridge, 2004.

[4] H. Chen and F. Allgöwer. A quasi-infinite horizon nonlinear model predictive control scheme with guaranteed stability. Automatica, 34(10):1205-1217, 1998.

[5] T. Faulwasser, B. Kern, and R. Findeisen. Model predictive pathfollowing for constrained nonlinear systems. In Proc. 48th IEEE Conf. on Decision and Control held jointly with the 2009 28th Chinese Control Conf. CDC/CCC 2009, pages 8642-8647, December 15-18, 2009.

[6] F. Fontes. A general framework to design stabilizing nonlinear model predictive controllers. Sys. Contr. Lett., 42(2):127-143, 2001.

[7] M. Henson and D. Seborg. Nonlinear Process Control. Prentice Hall, Upper Saddle River, NJ, 1997.

[8] B. Kern, C. Böhm, R. Findeisen, and F. Allgöwer. Receding horizon control for linear periodic time-varying systems subject to input constraints. In L. Magni, D. Raimondo, and F. Allgöwer, editors, Nonlinear Model Predictive Control - Towards New Challenging Applications, volume 384 of Lecture Notes in Control and Information Sciences, pages 109-117. Springer Berlin, 2009.

[9] L. Magni, G. De Nicolao, and R. Scattolini. Output feedback and tracking of nonlinear systems with model predictive control. Automatica, 37(10):1601-1607, 2001.

[10] L. Magni and R. Scattolini. Tracking of non-square nonlinear continuous time systems with piecewise constant model predictive control. J. Pro. Contr., 17(8):631-640, 2007.

[11] D.Q. Mayne, J.B. Rawlings, C.V. Rao, and P.O.M. Scokaert. Constrained model predictive control: Stability and optimality. Automatica, 36(6):789-814, 2000.

[12] H. Michalska. Trajectory tracking control using the receding horizon strategy. In Symposium on Control, Optimization and Supervision: CESA '96 IMACS, 1996.

[13] V.N. Phat and V. Jeyakumar. Stability, stabilization and duality for linear time-varying systems. Optimization, 59(4):447-460, 2010. 